\title{
Proton-implanted sapphire planar and channel waveguides
}

\author{
L. Laversenne, A. Crunteanu, P. Hoffmann, M. Pollnau \\ Applied Photonics Laboratory, Institute of Biomedical Imaging, Optics, and Engineering, \\ Swiss Federal Institute of Technology, CH-1015 Lausanne, Switzerland \\ P. Moretti, J. Mugnier \\ Physical Chemistry of Luminescent Materials, UMR CNRS 5620 \\ Claude Bernard-Lyon 1 University, F-69622 Villeurbanne Cedex, France
}

Implantation of $\mathrm{H}^{+}$and $\mathrm{He}^{+}$ions is particularly suitable to produce optical waveguides since these light ions produce a buried damage layer caused by nuclear collision at the end of the ion tracks. In the case of crystals, a low-refractive-index barrier is formed and the area between the barrier and the surface acts as a waveguide [1,2]. In this work, we demonstrate the first planar waveguides fabricated by proton implantation into sapphire $\left(\alpha-\mathrm{Al}_{2} \mathrm{O}_{3}\right)$ and the feasibility of channel waveguide formation by polyimide strip-loading of the samples.

The polished substrates $\left[\alpha-\mathrm{Al}_{2} \mathrm{O}_{3}(0001)\right]$ were irradiated with protons by use of a Van de Graff accelerator with low beam currents. The $\mathrm{H}^{+}$energy was $1.1 \mathrm{MeV}$ with fluences in the range $2-8 \times 10^{16} \mathrm{H}^{+} / \mathrm{cm}^{2}$. A qualitative investigation of the guiding properties of the implanted samples was performed by end-coupling a fundamental-mode laser beam into the guiding plane and recollimating the beam out-coupled from the rear end by employing microscope objectives. Surprisingly, our implanted samples exhibit good planar-waveguide properties even without annealing. The field pattern of the waveguide modes recorded with a CCD camera clearly showed a good confinement of the excited modes in the guiding region for all four different implantation doses (see Fig. 1a). Channel waveguides were then created by a polyimide spin-coating onto the irradiated surface followed by laser micro-structuring of the overlay. With the additional light confinement in the horizontal direction, these samples show channel waveguiding (see Fig. 1b).

The guiding properties of the ion-implanted planar waveguides were investigated using the dark m-lines technique by focusing a laser beam $(\lambda=632.8 \mathrm{~nm})$ onto the sample through a coupling prism. After determining the effective indeces of the giuded modes, the refractive-index profile was reconstructed using the inverse WKB method. Our measurements demonstrate that with the four doses investigated low-transverse-mode waveguides of good quality are formed. This is confirmed by the thinness of the dark lines observed for both, TM and TE modes. Figure 2 shows the reconstructed refractive-index profile of the TM modes in $\mathrm{H}^{+}$implanted sapphire (fluence: $8 \times 10^{16} \mathrm{H}^{+} / \mathrm{cm}^{2}$ ) where 6 propagating modes were observed. The barrier region is located at approximately $9 \mu \mathrm{m}$ depth and is characterized by a decrease in refractive index equal to $\Delta \mathrm{n}=1.3 \%$. The depth of the barrier is in reasonable agreement with the ion-range and damage-peak distributions computed from the SRIM code. The calculated $\mathrm{H}^{+}$range was $10.2 \mu \mathrm{m}$. The observed difference could be related to an enlargement of the damaged and stressed area not considered in the simulation for such high implantation doses.

The present results are very promising, since good guiding properties have been obtained even without annealing of the sample. The waveguides implanted so far are multi-mode, but adjustment of the $\mathrm{H}^{+}$energy and, therefore, the implantation depth will allow for the fabrication of fundamental-mode waveguides. Multi-energy implantation may increase the width of the damaged barrier and lead to an even better confinement of the modes.

Possible applications of passive sapphire waveguides include interferometry (Mach-Zehnder set-up) and high-power applications that exploit the superior thermo-mechanical and thermo-optical properties of sapphire. One can also transfer the present results to $\mathrm{Ti}^{3+}$-doped sapphire crystals to obtain active waveguides and possibly channel-waveguide lasers.

[1] P.D. Townsend, P.J. Chandler, L. Zhang, "Optical effects of ion implantation", P.L. Knight, A.Miller ed. (Cambridge University Press, Cambridge, 1994).

[2] P. Moretti, P. Thevenard, K. Wirl, P. Hertel, H. Hesse, E. Krätzig, G. Godefroy, "Proton implanted waveguides in $\mathrm{LiNbO}_{3}, \mathrm{KNbO}_{3}$ and $\mathrm{BaTiO}_{3}$ ", Ferroelectics 128, 13-18 (1992).

a)

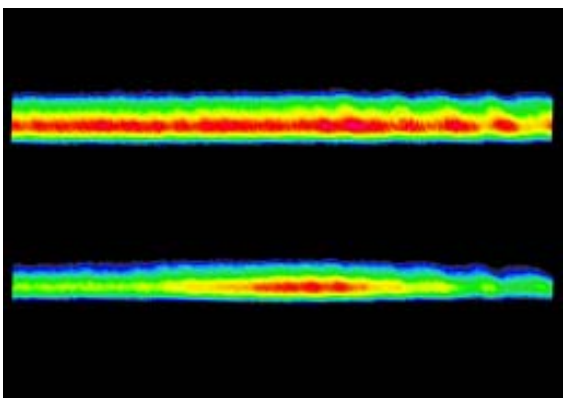

Fig. 1. Optical output profile of end-coupled, fundamental-mode laser light at $\lambda=785 \mathrm{~nm}$ from the guiding region of sapphire implanted by $8 \times 10^{16} \mathrm{H}^{+} / \mathrm{cm}^{2}$ with $1.1 \mathrm{MeV}$ (a) planar region, (b) under-rib region

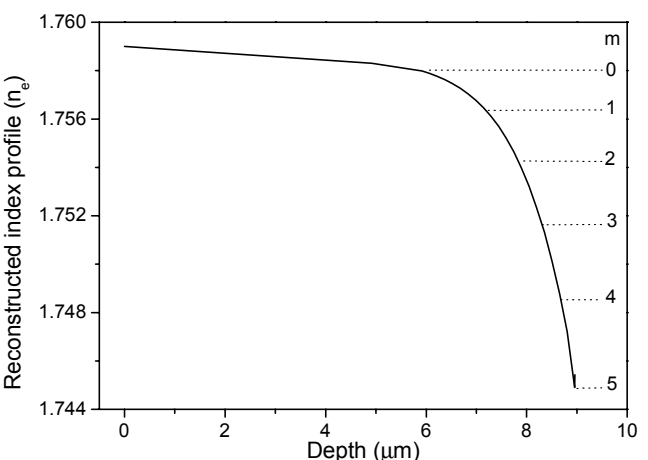

Fig. 2. Waveguide refractive-index profile for $8 \times 10^{16} \mathrm{H}^{+} / \mathrm{cm}^{2}$ with 1.1 $\mathrm{MeV}$, reconstructed from a dark $\mathrm{m}$-lines measurement. The dark $\mathrm{m}$-lines corresponding to the 6 guided modes are indicated at the right-hand side 
Abstract:

We report low-order transverse-mode planar waveguides in sapphire fabricated for the first time by proton implantation. The waveguides show good guiding properties without post-implantation annealing. Channel waveguiding was achieved by polyimide strip-loading. 